Canad. Math. Bull. Vol. 20 (1), 1977

\title{
IRRATIONAL NUMBERS ARISING FROM CERTAIN DIFFERENTIAL EQUATIONS
}

\author{
BY
}

M. RAM MURTY AND V. KUMAR MURTY

Niven [3] gave a simple proof that $\pi$ is irrational. Koksma [2] modified Niven's proof to show that $e^{r}$ is irrational for every non-zero rational $r$. Dixon [1] made a similar modification to show that $\pi$ is not algebraic of degree 2. In this note, we prove a general theorem which gives Niven's and Koksma's results as easy corollaries. A suitable modification in our proof also gives Dixon's result.

THEOREM 1. Let $G$ be non-trivial solution of the equation

$$
L(u)=p_{0} u^{(n)}+p_{1} u^{(n-1)}+\cdots+p_{n} u=0
$$

where $p_{i}$ are rational numbers and $p_{n} \neq 0$. If $b>0$ is such that $G(x) \geq 0$ on $[0, b]$ and $G^{(i)}(0), G^{(i)}(b)$ are rational for $0 \leq i \leq n-1$, then $b$ is irrational.

Proof. Without any loss of generality, we may suppose that the $p_{i}$ are integers. Suppose $b$ is rational and set $b=p / q,(p, q)=1, p, q \in \mathbb{Z}$. Set $f_{m}(x)=$ $1 / m !(q x)^{m}(p-q x)^{m}$, where $m$ is a natural number. It is easy to see that $f_{m}^{(k)}(0)$ are integers for $k \geq 0$ and since $f_{m}(x)=f_{m}(b-x)$, the same is true of $f_{m}^{(k)}(b)$. Now define the sequence $\left\{t_{k}\right\}$ recursively as follows:

$$
\begin{gathered}
t_{0}=1, \\
p_{n} t_{1}-p_{n-1} t_{0}=0, \\
p_{n} t_{2}-p_{n-1} t_{1}+p_{n-2} t_{0}=0, \\
p_{n} t_{n-1}-p_{n-1} t_{n-2}+\cdots+(-1)^{n-1} p_{1} t_{0}=0, \\
p_{n} t_{n \div r}-p_{n-1} t_{n+r-1}+\cdots+(-1)^{n} p_{0} t_{r}=0 \text { for } r \geq 0 .
\end{gathered}
$$

Clearly, $p_{n}^{k} t_{k}$ is an integer for $k \geq 0$. Let

$$
F_{m}(x)=\sum_{r=0}^{2 m} t_{r} f_{m}^{(r)}(x)
$$

Received by the editors Sept. 15, 1976. 
If $L^{*}$ is the adjoint of $L$, we have

$$
\begin{aligned}
L^{*}\left(F_{m}(x)\right) & =\sum_{k=0}^{n}(-1)^{k} p_{n-k} F_{m}^{(k)}(x) \\
& =\sum_{k=0}^{n}(-1)^{k} p_{n-k} \sum_{r=0}^{2 m} t_{r} f_{m}^{(r+k)}(x) \\
& =\sum_{s=0}^{2 m} f_{m}^{(s)}(x) \sum_{r+k=s}(-1)^{k} p_{n-k} t_{r}=p_{n} f_{m}(x) .
\end{aligned}
$$

Letting

$$
\begin{aligned}
P(u, v)= & u\left[p_{n-1} v-\frac{d}{d x}\left(p_{n-2} v\right)+\cdots+(-1)^{n-1} \frac{d^{n-1}}{d x^{n-1}}\left(p_{0} v\right)\right]+ \\
& \frac{d u}{d x}\left[p_{n-2} v-\frac{d}{d x}\left(p_{n-3} v\right)+\cdots+(-1)^{n-2} \frac{d^{n-2}}{d x^{n-2}}\left(p_{0} v\right)\right]+ \\
& +\cdots+\frac{d^{n-1} u}{d x^{n-1}}\left(p_{0} v\right),
\end{aligned}
$$

we have by Lagrange's identity,

$$
F_{m}(x) L(G)-G(x) L^{*}\left(F_{m}(x)\right)=\frac{d}{d x} P\left(G, F_{m}\right),
$$

so that

$$
-\int_{0}^{b} p_{n} f_{m}(x) G(x) d x=\left[P\left(G, F_{m}\right)\right]_{0}^{b}
$$

since $L(G)=0$. As $p_{n}^{k} t_{k}$ is an integer, it follows that $p_{n}^{2 m} F_{m}^{(w)}(x)$ is an integer for $x=0$ and $b, w \geq 0$. Thus, if $A$ denotes the products of the denominators of $G^{(i)}(0)$ and $G^{(i)}(b), \quad 0 \leq i \leq n-1$ (when expressed in lowest terms), $A p_{n}^{2 m}\left[P\left(G, F_{m}\right)\right]_{0}^{b}$ is an integer for every $m$. Now

$$
A p_{n}^{2 m}\left[P\left(G, F_{m}\right)\right]_{0}^{b}=-A p_{n}^{2 m+1} \int_{0}^{b} f_{m}(x) G(x) d x .
$$

If $B$ and $C$ are such that $|G(x)| \leq B,|q x(p-q x)| \leq C$ on $[0, b]$ we have

$$
0<A p_{n}^{2 m+1}\left|\int_{0}^{b} f_{m}(x) G(x) d x\right|<\frac{b B A p_{n}^{2 m+1} C^{2 m}}{m !} .
$$

If $m$ is sufficiently large, the right hand side is $<1$, giving a contradiction. Hence $b$ is irrational.

COROllaRy. (1) $\pi^{2}$ is irrational, (hence so also is $\pi$ ). (2) $\log r$ is irrational for every rational $r>0, r \neq 1$. (3) $e^{r}, \sin r, \cos r, \cosh r \sinh r$ are irrational for every non-zero rational $r$. 
Proof. If $\pi^{2}$ is rational, consider $y^{\prime \prime}+\pi^{2} y=0$ which has as a solution $(1 / \pi) \sin$ $\pi x$. For $b=1$, we get a contradiction. This proves (1). (2) and (3) are proved similarly, using the equation $y^{\prime}-y=0$ or $y^{\prime \prime} \pm y=0$.

The following theorem is more arithmetical in nature.

Theorem 2. Let $G$ be a non-trivial solution of $y^{(n)}+t y=0$ where $t=$ $(u / v),(u, v)=1$, is a non-zero rational. Suppose $G^{(i)}(0)$ is rational for $0 \leq i \leq$ $n-1$ and for some $r \neq 0$ with $(r, n)=1$, we have $G^{(r)}(0) \neq 0$. If $\beta$ is a non-zero rational, then $G^{(n-1)}(\beta)$ is irrational.

Proof. Let $\beta=(a / b),(a, b)=1$. Define

$$
f_{p}(x)=\frac{(\beta-x)^{n p}\left[\beta^{n}-(\beta-x)^{n}\right]^{p-1} b^{n p+(n-1)(p-1)}}{(p-1) !},
$$

where $p$ is a prime soon to be specified. If we compute the $t_{k}$ in Theorem 1 for the equation $y^{(n)}+t y=0$, we find $t_{k}=0$ if $k \neq \equiv 0(\bmod n)$ and in case $k=$ $s n, t_{s n}=(-1)^{s n-s} t^{s}$. If we set

$$
F_{p}(x)=\sum_{k=0}^{M} t_{k} f_{p}^{(k)}(x)
$$

where $M=n(2 p-1)$, we have as in Theorem $1, L^{*}\left(F_{p}(x)\right)=t f_{p}(x)$, where $L^{*}$ is the adjoint of $L(y)=y^{(n)}+t y$. Since $f_{p}$ is a polynomial of degree $M, f_{p}^{(k)}(x) \equiv 0$ for $k>M$. If we set $\beta-x=y$ and $g_{p}(y)=y^{n p}\left(\beta^{n}-y^{n}\right)^{p-1}$, then

$$
g_{p}(y)=\sum_{i=0}^{p-1}(-1)^{i} \beta^{n(p-1-i)}\left(\begin{array}{c}
p-1 \\
i
\end{array}\right) y^{n(p+i)}
$$

from which it follows at once that $f_{p}^{(k)}(\beta)=0$ for all $k \neq n(p+i), 0 \leq i \leq p-1$ and

$$
f_{p}^{(k)}(\beta)=(-1)^{n(p+i)+i} \frac{b^{M-p+1}}{(p-1) !} \beta^{n(p-1-i)}\left(\begin{array}{c}
p-1 \\
i
\end{array}\right)[n(p+i)] !
$$

for $k=n(p+i)$. Hence $v^{2 p-1} F_{p}(\beta)$ is an integer divisible by $p$. Since $f_{p}$ has a zero of order $p-1$ at $x=0$, we have $f_{p}^{(k)}(0)=0$ for $k<p-1$. Writing $f_{p}(x)=$ $\left(x^{p-1} /(p-1) !\right) h_{p}(x)$ we see from $(p-1) ! f_{p}^{(k)}(x)=\sum_{s=0}^{k}\left(\begin{array}{l}k \\ s\end{array}\right)\left[x^{p-1}\right]^{(s)}\left[h_{p}(x)\right]^{(k-s)}$ that $f_{p}^{(k)}(0)=\left(\begin{array}{c}k \\ p-1\end{array}\right) h_{p}^{(k-p+1)}(0)$ for $k \geq p-1$. Clearly $f_{p}^{(k)}(0)$ is an integer as $h_{p}^{(k-p+1)}(0)$ is an integer. Also $\left(\begin{array}{c}k \\ p-1\end{array}\right)$ is divisible by $p$ if $k \geq p$, and $k \neq-1(\bmod$ $p)$. If $k \geq p$ and $k-p+1 \equiv 0(\bmod p)$, then $h_{p}^{(k-p+1)}(0)$ is divisible by $p$. If $k=p-1, f_{p}^{(p-1)}(0)=n^{p-1} a^{n p+(n-1)(p-1)}$. Hence, $f_{p}^{(k)}(0)$ is divisible by $p$ unless $k=p-1$. As $(r, n)=1$, let $p$ be a prime $>n a$, congruent to $-r(\bmod n)$. As $G^{(r)}(0) \neq 0$, and $p-1 \equiv n-r-1(\bmod n)$, the term $f_{p}^{(p-1)}(0)$ occurs once and 
only once in $\sum_{\mathrm{k}=0}^{\mathrm{n}-1}(-1)^{k} G^{(k)}(0) F_{p}^{(n-k-1)}$ and that is in the expression for $F_{p}^{(n-r-1)}(0)$. Let $N$ be the product of all the denominators of the rationals $G(0)$, $G^{\prime}(0), \ldots, G^{(n-1)}(0), G^{(n-1)}(\beta)$. (Here, we are supposing $G^{(n-1)}(\beta)$ is rational and will arrive at a contradiction). Thus, if $p>\max \left(n a, N G^{(r)}(0), u v\right)$, all terms in

$$
N v^{2 p-1}\left\{G^{(n-1)}(\beta) F_{p}(\beta)-\sum_{k=0}^{n-1}(-1)^{k} G^{(k)}(0) F_{p}^{(n-k-1)}(0)\right\}
$$

are divisible by $p$ except one term (the one involving $G^{(r)}(0) \neq 0$ ). Now, as in the proof of Theorem 1,

$$
\begin{aligned}
-u N v^{2 p} \int_{0}^{\beta} G(x) f_{p}(x) d x=N v^{2 p-1}\left\{G^{(n-1)}(\beta)\right. & \\
& \left.\times F_{p}(\beta)-\sum_{k=0}^{n-1}(-1)^{k} G^{(k)}(0) F_{p}^{(n-k-1)}(0)\right\} .
\end{aligned}
$$

Thus, it follows $u N v^{2 p} \int_{0}^{\beta} G(x) f_{p}(x) d x \neq o$ for an infinity of primes $p$, using Dirichlet's theorem. On the other hand, we know $u N v^{2 p} \int_{0}^{\beta} G(x) f_{p}(x) d x$ is an integer. This is a contradiction since

$$
\lim _{p \rightarrow \infty}\left|u N v^{2 p} \int_{0}^{\beta} G(x) f_{p}(x) d x\right|=0 .
$$

This proves the theorem.

COROLlary. Let $p$ be an odd prime and $G$ a non-trivial solution of $y^{(p)}+t y=$ $0, t$ a non-zero rational. If $G(0), \ldots, G^{(p-1)}(0)$ are rational and at least two of them are non-zero, then $G(\beta), G^{\prime}(\beta), \ldots, G^{(p-1)}(\beta)$ are irrational for any non-zero rational $\beta$.

Remark. The case $p=2$ has been covered by a corollary of Theorem 1 .

Proof. As at least two of $G(0), G^{\prime}(0), \ldots, G^{(p-1)}(0)$ are non-zero, there is an $r$ such that $G^{(r)}(0) \neq 0$ and $(r, p)=1$. The conditions of the theorem are satisfied and so $G^{(p-1)}(\beta)$ is irrational. As $G^{(i)}(x)$ also satisfies the conditions of the theorem for $0<i \leq p-1$ the result follows.

Acknowledgement. We would like to thank Professor P. R. Beesack for his helpful suggestions.

\section{REFERENCES}

1. J. D. Dixon, $\pi$ is not algebraic of degree one or iwo, Amer. Math. Monthly, 69 (1962), 632.

2. J. F. Koksma, On Niven's proof that $\pi$ is irrational, Nieuw Archief voor Wiskunde, (2) 23 (1949), 39.

3. I. Niven, A simple proof that $\pi$ is irrational, Bull. Amer. Math. Soc., 53 (1947), 509.

DePARTMENT OF MATHEMATICS

CARLETON UNIVERSITY

OtTawa, OnTARio 